\title{
Genetic Correlations Between Brain Volumes and the WAIS-III Dimensions of Verbal Comprehension, Working Memory, Perceptual Organization, and Processing Speed
}

\author{
Daniëlle Posthuma', Wim F. C. Baaré2, Hilleke E. Hulshoff Pol'², René S. Kahn², Dorret I. Boomsma', \\ and Eco J. C. De Geus' \\ ' Department of Biological Psychology,Vrije Universiteit Amsterdam, The Netherlands \\ ${ }^{2}$ Department of Psychiatry, University Medical Centre, Utrecht, The Netherlands
}

W e recently showed that the correlation of gray and white matter volume with full scale $\mathrm{IQ}$ and the Working Memory dimension are completely mediated by common genetic factors (Posthuma et al., 2002). Here we examine whether the other WAIS III dimensions (Verbal Comprehension, Perceptual Organization, Processing Speed) are also related to gray and white matter volume, and whether any of the dimensions are related to cerebellar volume. Two overlapping samples provided 135 subjects from 60 extended twin families for whom both MRI scans and WAIS III data were available. All three brain volumes are related to Working Memory capacity $(r=0.27)$. This phenotypic correlation is completely due to a common underlying genetic factor. Processing Speed was genetically related to white matter volume $\left(r_{g}=0.39\right)$. Perceptual Organization was both genetically $\left(r_{g}=0.39\right)$ and environmentally $\left(r_{\mathrm{e}}=-0.71\right)$ related to cerebellar volume. Verbal Comprehension was not related to any of the three brain volumes. It is concluded that brain volumes are genetically related to intelligence which suggests that genes that influence brain volume may also be important for intelligence. It is also noted however, that the direction of causation (i.e., do genes influence brain volume which in turn influences intelligence, or alternatively, do genes influence intelligence which in turn influences brain volume), or the presence or absence of pleiotropy has not been resolved yet.

Two independent studies recently quantified the contribution of genetic and environmental factors to interindividual differences in brain volumes (Baaré et al., 2001; Thompson et al., 2001). Baaré et al. (2001) used magnetic resonance imaging (MRI) to measure intracranial space, total brain volume, total white matter volume, total gray matter volume, and lateral ventricle volume in 258 subjects belonging to 112 (extended) twin families. They reported very high heritabilities for all volumes (ranging from 80 to $90 \%)$ except ventricular volume (no genetic influences). Thompson et al. (2001) used voxel based MRI techniques on $10 \mathrm{MZ}$ twin pairs and $10 \mathrm{DZ}$ twin pairs and reported high heritability of gray matter volume in several cortical regions. Results from previous studies also suggested that genetic factors are much more important than environmental factors for inter-individual differences in brain volumes (Bartley et al., 1997; Carmelli et al., 1998; Carmelli et al., 2002; Pennington et al., 2000; Reveley et al., 1984).

Since the second half of the 19th century positive correlations between head size (as measured with a measuring tape around the head) and psychometric intelligence have been observed. Correlations generally range around 0.20 (Jensen, 1994; Posthuma et al., 2001a), but can be as high as 0.44 (van Valen, 1974). MRI provides a more accurate measure of the size of the brain, as head size includes both brain volume and thickness of the skull. Several studies have correlated MRI-brain volumes with measures of intelligence, and, on average, brain volume as measured with MRI and IQ correlate around 0.40 (e.g., Andreasen et al., 1993; Egan et al., 1994; Raz et al., 1993; Storfer, 1999; Wickett et al., 2000; Willerman et al., 1992).

Three multivariate genetic studies of brain volume and intelligence have investigated the nature of the correlation between brain volume and intelligence (Pennington et al., 2000; Thompson et al., 2001; Wickett et al., 1997), but did not have the optimal design (Pennington et al., 2000; Wickett et al., 1997) or enough statistical power (Thompson et al., 2001) to decompose the observed correlation into genetic and environmental components. Using a dataset consisting of $24 \mathrm{MZ}$ pairs, $31 \mathrm{DZ}$ pairs, and 25 additional siblings ( 135 individuals from 60 families) for whom both data on brain volume and intelligence were available, we recently showed that the correlations between gray or white matter volume to full scale IQ (WAIS III) and its Working Memory dimension are completely mediated by an underlying set of genes that influences both brain volumes and IQ (Posthuma et al., 2002).

Address for correspondence: D. Posthuma, Vrije Universiteit, Department of Biological Psychology, van der Boechorststraat 1, 1081 BT, Amsterdam, the Netherlands, Email: danielle@psy.vu.nl 
Using the same sample of 135 individuals from 60 families we now investigate whether three different brain volumes (total white matter of the cerebrum, total gray matter of the cerebrum, total volume of the cerebellum) are differentially correlated to each of the other standard WAIS III dimensions: Verbal Comprehension, Perceptual Organization, and Processing Speed. We expect to find differential correlations between each of the three brain volumes and each of the four WAIS III dimensions. For example, the WAIS III dimension Processing Speed is an index of the speed of central nervous system processing (WAIS III, 1997), and is therefore expected to be related strongest to white matter volume, as white matter reflects the degree of interconnectiveness between neuronal cells.

The three brain volumes were obtained by using MR imaging in a large Dutch sample (258 Dutch adults from 112 extended twin families) of twins and their siblings (Baaré et al., 2001). Using structural equation modelling on the combined MRI and WAIS III datasets, we will test whether the correlation between these brain volumes and WAIS III dimensions is genetically or environmentally mediated.

\section{Methodology \\ Subjects - WAIS III Sample}

Six hundred eighty-eight family members from 271 extended twin families participated in an ongoing study on the genetics of adult brain function (Posthuma et al., 2001a, 2001b; Posthuma, 2002; Wright et al., 2001) until December 2000. All participants were obtained from the Netherlands Twin Registry (Boomsma, 1998). Zygosity was determined by DNA fingerprinting. The complete sample consisted of two age cohorts: a young adult cohort with a mean of 26.2 years of age $(S D=4.19)$ and an older adult cohort with a mean around 50.4 years of age $(S D=7.51)$. Participating families consisted of one to eight siblings (including twins). On average 2.5 subjects per family participated. In the young cohort 171 males and 210 females participated, in the older cohort 135 and 172 respectively. The young cohort included $54 \mathrm{MZ}$ pairs, $73 \mathrm{DZ}$ pairs, 18 single twins and 109 additional siblings. The older cohort included $48 \mathrm{MZ}$ pairs, $58 \mathrm{DZ}$ pairs, 15 single twins, and 80 additional siblings (for a detailed description of the sample characteristics see Posthuma et al., 2001b). The study was approved by the scientific and ethical committee of the Vrije Universiteit Amsterdam. Subjects were paid NLG 50 (23 EUROS) for participation.

\section{Subjects - MRI sample}

The MRI sample was obtained from a large study on the genetics of brain volumes (Baaré et al., 2001; Posthuma et al., 2000). For this second dataset, subjects were recruited from the (healthy) twin sample of the department of Psychiatry of the University Medical Center Utrecht, the Netherlands, and from the Netherlands Twin Registry. One hundred and twelve pairs of twins (112 families), $33 \mathrm{MZ}$ male (MZM), 17 DZ male (DZM), $21 \mathrm{MZ}$ female (MZF), 20 DZ female (DZF), and 21 DZ opposite-sex (DOS), and 19 male (SM) and 15 female (SF) full siblings participated in the study. Zygosity was determined by DNA fingerprinting.
Subjects were required not to have any severe medical diseases. Mental and physical health was assessed by means of the Family Interview for Genetic Studies (Nurnberger et al., 1994), and a medical history inventory, respectively. All subjects gave written informed consent to participate in the study after full explanation of the study aims and procedures. The study was approved by the scientific and ethical committee of the University Medical Center Utrecht. Subjects were paid NLG 75 (34 EUROS) for participation.

\section{Overlap Between the Two Samples}

The combined dataset consisted of 808 subjects from 322 families. For 135 subjects from 60 families data on both IQ and MRI-scans were available. This "overlapping" dataset consisted of 16 families from which MZ twins (without additional non-twin siblings) participated, 8 families from which the MZ twins and one additional sibling participated, 21 families from which the DZ twins (without additional non-twin siblings) participated, 10 families from which the DZ twins and one additional sibling participated, two families from which one twin and one non-twin sibling participated, and three families from which only one member participated. In other words, the overlapping dataset consisted of $24 \mathrm{MZ}$ twins and $69 \mathrm{DZ}$ twins/sib pairs. The mean age in the overlapping dataset was 29.2 $(S D=7.34)$. There were 57 females and 78 males.

To obtain the most accurate estimates of means and variances of IQ scores and brain volumes, the combined dataset of 808 subjects was used in the analyses, as opposed to using only the subset with data on both MRI and WAISIII scores. In Mx (Neale, 1997) such incomplete datasets can be handled easily (see also Statistical Analyses).

The average time between the MRI scans and the IQ measurements was 13 weeks, ranging from -1.5 years (IQ measurement before MRI scans) to + 3.3 years (MRI scan before IQ measurement). Age at time of IQ measurement was included as an effect on the IQ scores while age at time of the MRI scans was included as an effect on brain volumes.

\section{Intelligence Testing}

Psychometric IQ was measured with the Dutch adaptation of the WAIS III (WAIS-III, 1997). As we previously showed (Posthuma et al., 2001b) that our IQ sample is representative of the Dutch population and the present sample size exceeds the WAIS III standardization sample, we report unstandardized raw IQ scores, and explicitly model the effects of sex and age in the multivariate analysis. Individual scores for each subtest except digit-symbol substitution were calculated by weighting the observed score by the maximum possible score on that subtest times 100 (i.e., percentage correct on each subtest). For digit-symbol substitution the number of correct items per 60 seconds was calculated. Nine subtests were used to calculate the four dimensions according to the WAIS III guidelines (1997); Verbal Comprehension (VC; the mean percentage correct of subtests information, similarities, and vocabulary), Working Memory (WM; the mean percentage correct of subtests arithmetic and letter-number sequencing), Perceptual Organization (PO; the mean percentage correct of subtests block design, matrix reasoning, and picture 
completion), and Processing Speed (PS; the number of correct items per 60 seconds of subtest digit-symbol substitution). The validity of these four dimensions was recently confirmed by a reanalysis of the WAIS manual data by Deary (2001).

\section{MR Image Acquisition and Processing}

MR images were obtained on a 1.5 Tesla Philips Gyroscan scanner at the University Medical Center Utrecht. For volumetric analysis a three dimensional (3D) T1 — weighted, coronal, spoiled gradient echo scan (FFE) of the whole head $\left(\mathrm{TE}=4.6 \mathrm{~ms}, \mathrm{TR}=30 \mathrm{~ms}\right.$, flip angle $=30^{\circ}, 170-180$ contiguous slices; $1 \times 1 \times 1.2 \mathrm{~mm}^{3}$ voxels), and a coronal dual contrast turbo spin echo (DTSE) of the whole brain $(\mathrm{TE} 1=14 \mathrm{~ms}, \mathrm{TE} 2=80 \mathrm{~ms}, \mathrm{TR}=6350 \mathrm{~ms}, 120$ contigu ous slices; $1 \times 1 \times 1.6 \mathrm{~mm}^{3}$ voxels) were acquired.

Images were coded to ensure blindness for subject identification, zygosity and family membership. Image volumes were transformed into Talairach space (no scaling) (Talairach \& Tournoux, 1988) and corrected for magnetic field inhomogeneities. Volumetric measurements were obtained using automated segmentation procedures and included intracranial, whole brain, gray and white matter of the cerebrum (excluding cerebellum and brain stem), and lateral and third ventricle volumes (Schnack et al., 2001a; Schnack et al., 2001b). Automatic segmentation software included histogram analysis algorithms, anatomical knowledge based decision rules and series of mathematical morphological operators to connect all voxels of interest. Intracranial volume was segmented on DTSE scans. Cerebral gray and white matter volumes were obtained after cerebellar and brain stem tissue was removed. The segmentation procedures yielded highly reliable volume measurements with inter-rater intraclass correlations all above 0.96 .

The present study included gray matter volume of the cerebrum, white matter volume of the cerebrum and cerebellar volume. Cerebellar volume was not separated into white and gray matter volumes as the location of the cerebellum complicates the reliable separation of cerebellar white and gray matter volume (i.e., at the edges of the coil artefacts will influence this separate detection). However, the detection of total cerebellar volume does not suffer from these artefacts and can be reliably measured.

\section{Statistical Analyses}

As the sample consisted of unbalanced pedigrees and had some missing data, models were fitted to the raw data instead of covariance matrices. This was accomplished by using the rectangular data file option in Mx (Neale, 1997).

We previously determined whether interindividual variation in each of the four WAIS III dimensions could be explained by additive genetic influences (A), dominance genetic influences (D), shared environmental influences (C), or non-shared environmental influences (E). We found that shared environmental influences on each of these dimensions were non-significantly different from zero, and that all four dimensions were highly heritable (ranging from $66 \%$ to $83 \%$ ) (Posthuma et al., 2001a), in line with estimates from previous studies (Bouchard \& McGue, 1981; McClearn et al., 1997). Heritability estimates did not differ across males and females, but cohort differences existed for Working Memory. In the young cohort the genetic variation was mainly due to dominance genetic variation whereas in the older cohort the genetic variation was additive. The broad heritability estimates of the Working Memory dimension, however, were homogeneous across cohorts.

For the MRI measures as well as for the IQ measures shared environmental influences were non-significantly different from zero (Baaré et al., 2001; Posthuma et al., 2000; Posthuma et al., 2001a) and a model that decomposed the variance in genetic variance $(\mathrm{A})$ and non-shared environmental variance (E) fitted well for all brain volumes and WAIS III dimensions. Therefore, the (co-)variances in the multivariate genetic models were decomposed into two possible latent sources of variance: genetic variance (A), and non-shared environmental variance (E). The latter also includes all sources of variance due to measurement error. For DZ twin pairs (and sibpairs) similarity of addixtive genetic influences was set at 50\%, and no similarity in non-shared environmental influences. For MZ twin pairs, similarity of additive genetic variance was set at $100 \%$ and similarity in nonshared environmental influences was fixed at zero.

\section{Using Structural Equation Modelling to Decompose} the (co-)variance into Genetic and Environmental Components

Decomposition of the variances and covariances into genetic (A) and environmental (E) components was obtained using structural equation modelling with maximum likelihood estimation. Environmental factors incorporate those factors in the environment that are not shared by siblings. Let matrices $\mathrm{A}$ and $\mathrm{E}$ be symmetric and of dimensions $7 \times 7$ (for seven variables; total gray matter, total white matter, cerebellar volume, Verbal Comprehension, Working Memory, Perceptual Organization, and Processing Speed). Matrix A denotes the genetic component while matrix $\mathrm{E}$ denotes the environmental component. The diagonal elements of matrix A denote the genetic variances of each of the seven variables. For example, element $a_{11}$ is the genetic variation in gray matter volume. The off-diagonal elements of matrix A represent the genetic covariance between variables. Analogously, the diagonal elements of matrix E denote the environmental variances of the seven variables, and the off-diagonal elements denote the covariances due to environmental influences.

As matrices $\mathrm{A}$ and $\mathrm{E}$ are covariance matrices, they are restricted to be positive definite. This is accomplished by calculating matrix $\mathrm{A}$ and $\mathrm{E}$ as the product of a triangular matrix and its transpose. Thus, matrix $\mathrm{A}$ is calculated as $\mathrm{X} \times \mathrm{X}$, where $\mathrm{X}$ is triangular and of dimensions $7 \times 7$ (for seven variables). Analogously, matrix $E$ is $Z \times Z$ '. This is also known as a Cholesky factorization of matrices $\mathrm{A}$ and $\mathrm{E}$.

The decomposition of variances and covariances into genetic and environmental components necessitates the use of a genetically informative design, such as the twin design. The correlation between the genetic component that influences the phenotype of one twin and the genetic component that influences the phenotype of the co-twin is 1 for $M Z$ twins and 0.5 for $\mathrm{DZ}$ twins/sibling pairs. The correlation between the environmental component that influences the phenotype of one twin and the environmental component 
that influences the phenotype of the co-twin is zero for both $\mathrm{MZ}$ and $\mathrm{DZ}$ twins/sibling pairs.

The variance is formally represented as

$$
A+E=X \times X^{\prime}+Z \times Z^{\prime} .
$$

The covariance is formally represented as

$$
\begin{array}{ll}
A=X \times X^{\prime} & \text { for MZ twins, } \\
0.5 \times A=0.5 \times X \times X^{\prime} & \text { for DZ twins. }
\end{array}
$$

The genetic correlation between variables $i$ and $j\left(a_{i j}\right)$ is derived as the genetic covariance $\left(a_{i j}\right)$ between variables $i$ and $j$ divided by the square root of the product of the genetic variances of variables $i\left(a_{i i}\right)$ and $j\left(a_{j j}\right)$ :

$$
r_{g i j}=\frac{a_{i j}}{\sqrt{a_{i i} \times a_{j j}}} .
$$

Analogously, the environmental correlation $\left(r_{e i j}\right)$ between variables $i$ and $j$ is derived as the environmental covariance between variables $i$ and $j$ divided by the square root of the product of the environmental variances of variables $i$ and $j$ :

$$
r_{e i j}=\frac{e_{i j}}{\sqrt{e_{i i} \times e_{j j}}} .
$$

The phenotypic correlation $(r)$ is the sum of the product of the genetic correlation and the square roots of the genetic variances of the two phenotypes and the product of the environmental correlation and the square roots of the environmental variances of the two phenotypes. Or, in other words, the phenotypic correlation is composed of a genetic contribution and an environmental contribution.

$$
r=r_{g i j} \times \sqrt{\frac{a_{i i}}{\left(a_{i i}+e_{i i}\right)}} \times \sqrt{\frac{a_{j j}}{\left(a_{j j}+e_{j j}\right)}}
$$

$$
+r_{e i j} \times \sqrt{\frac{e_{i i}}{\left(a_{i i}+e_{i i}\right)}} \times \sqrt{\frac{e_{j j}}{\left(a_{j j}+e_{j j}\right)}} .
$$

\section{Results \\ Descriptives}

Means, and standard deviations of the WAIS III scores clearly show that males generally have a higher score on the WAIS III dimensions, except on Processing Speed, where females are faster than males (Table 1a). These effects are evident in both the young and the older cohort. Subjects in the older cohort generally have a lower score on all four WAIS III dimensions (see Table 1a).

The MRI-data set was not divided in two age cohorts as the median of age was 28.6 years and $83 \%$ of the sample were younger than 36. Thus, for brain volumes, age (at time of MRI-scan) was included as a linear effect on the mean volumes. A sex difference in brain volume is evident in each of the three total volumes; males generally have larger volumes than females (see Table1b).

Table 2 presents the phenotypic correlations on the variables adjusted for the effects of sex, age or cohort.

The Working Memory dimension of the WAIS III consistently and significantly correlated to all three brain volumes. The Verbal Comprehension dimension did not correlate significantly with any of the brain volumes. Perceptual Organization correlated significantly with gray matter volume and cerebellar volume, but not with white matter volume. Processing speed correlates significantly with white matter volume, and the correlation with gray

\begin{tabular}{|c|c|c|c|c|c|}
\hline & Age & VC & WM & PO & PS \\
\hline \multicolumn{6}{|c|}{ Young Females } \\
\hline Mean & 26.0 & 63.6 & 62.2 & 79.9 & 44.0 \\
\hline$N$ & 210 & 210 & 210 & 210 & 208 \\
\hline$S D$ & 4.0 & 11.2 & 13.0 & 11.0 & 6.7 \\
\hline \multicolumn{6}{|c|}{ Young Males } \\
\hline Mean & 26.3 & 67.5 & 66.1 & 83.9 & 39.4 \\
\hline$N$ & 171 & 171 & 171 & 171 & 168 \\
\hline$S D$ & 4.4 & 12.6 & 13.2 & 9.4 & 6.4 \\
\hline \multicolumn{6}{|c|}{ Older Females } \\
\hline Mean & 50.5 & 58.1 & 54.2 & 66.1 & 35.7 \\
\hline$N$ & 172 & 172 & 172 & 172 & 172 \\
\hline$S D$ & 7.7 & 13.2 & 13.5 & 12.2 & 8.4 \\
\hline \multicolumn{6}{|c|}{ Older Males } \\
\hline Mean & 50.3 & 65.0 & 64.0 & 69.8 & 35.3 \\
\hline$N$ & 135 & 135 & 135 & 135 & 135 \\
\hline$S D$ & 7.3 & 13.0 & 12.4 & 12.7 & 6.3 \\
\hline
\end{tabular}
matter volume was almost significant $(p=0.07)$.

A seven-variate Cholesky decomposition of gray matter volume, white matter volume, cerebellar volume, Verbal Comprehension, Working Memory, Perceptual Organization, and Processing Speed was conducted to estimate the contributions of genetic factors and non-shared environmental

Table 1a

Descriptives of WAIS III Dimensions 


\section{Table 1b}

Descriptives of Brain Volumes

\begin{tabular}{lcccc}
\hline & Age & GMV (in $\left.\mathrm{cm}^{3}\right)$ & WMV (in $\left.\mathrm{cm}^{3}\right)$ & CBV (in $\left.\mathrm{cm}^{3}\right)$ \\
\hline Females & & & & \\
$\quad$ Mean & 31.7 & 612.4 & 422.9 & 133.6 \\
$N$ & 118 & 118 & 118 & 118 \\
$S D$ & 10.2 & 63.0 & 51.9 & 12.2 \\
Males & & & & \\
Mean & 30.6 & 668.2 & 493.8 & 147.0 \\
$N$ & 140 & 140 & 140 & 140 \\
$S D$ & 9.0 & 60.1 & 50.1 & 11.2 \\
\hline
\end{tabular}

Note: $\mathrm{GMV}=$ gray matter volume; $\mathrm{WMV}=$ white matter volume; $\mathrm{CBV}=$ cerebellar volume. $N=$ number of subjects; $S D=$ standard deviation .

\section{Table 2}

Pearson Correlations Between Gray Matter Volume, White Matter Volume, Cerebellar Volume, Verbal Comprehension, Working Memory, Perceptual Organization and Processing Speed. Individual Scores on Each Variable Are Adjusted for the Effects of Sex, Age and Cohort

\begin{tabular}{lllllll}
\hline \multicolumn{2}{c}{ GMV } & WMV & CBV & VC & WM & PO \\
\hline WMV & $0.59^{* *}$ & & & & & \\
CBV & $0.47^{* *}$ & $0.49^{* *}$ & & & & \\
VC & $\mathbf{0 . 0 6}$ & $\mathbf{0 . 0 1}$ & $\mathbf{0 . 0 3}$ & & & \\
WM & $\mathbf{0 . 2 7 ^ { * * }}$ & $\mathbf{0 . 2 8 ^ { * * }}$ & $\mathbf{0 . 2 7 ^ { * * }}$ & $0.54^{* *}$ & & \\
PO & $\mathbf{0 . 2 0 ^ { * }}$ & $\mathbf{0 . 0 8}$ & $\mathbf{0 . 1 8 ^ { * }}$ & $0.49^{* *}$ & $0.51^{* *}$ \\
PS & $\mathbf{0 . 1 6}$ & $\mathbf{0 . 2 5 ^ { * * }}$ & $\mathbf{0 . 1 1}$ & $0.28^{* *}$ & $0.40^{* *}$ & $0.34^{* *}$ \\
\hline Note: Intra-domain correlations Printed in normal text, Inter-domain correlations are \\
printed in bold. \\
* significant at the 0.05 level; ** significant at the 0.01 level. ( $N=258$ for brain \\
volumes, $N=135$ for inter-domain correlations; $N=688$ for WAIS III dimensions).
\end{tabular}

Table 3a

Genetic (Below Diagonal) and Environmental (Above Diagonal) Correlations and 95\% Confidence Intervals (in Brackets) from the AE Full Cholesky Model

\begin{tabular}{|c|c|c|c|c|c|c|c|}
\hline & GMV & WMV & CBV & VC & WM & PO & PS \\
\hline \multirow[t]{2}{*}{ GMV } & & 0.00 & 0.08 & -0.14 & -0.13 & 0.19 & 0.00 \\
\hline & & $(-0.24-0.25)$ & $(-0.17-0.32)$ & $(-0.43-0.18)$ & $(-0.38-0.15)$ & $(-0.10-0.46)$ & $(-0.27-0.28)$ \\
\hline \multirow[t]{2}{*}{ WMV } & 0.69 & & 0.35 & 0.07 & 0.03 & -0.22 & -0.17 \\
\hline & $(0.58-0.79)$ & & $(0.10-0.56)$ & $(-0.35-0.49)$ & $(-0.30-0.38)$ & $(-0.50-0.18)$ & $(-0.50-0.19)$ \\
\hline \multirow[t]{2}{*}{ CBV } & 0.49 & 0.47 & & -0.23 & -0.05 & -0.71 & 0.26 \\
\hline & $(0.35-0.62)$ & $(0.33-0.59)$ & & $(-0.58-0.22)$ & $(-0.34-0.29)$ & $(-0.84-0.35)$ & $(0.09-0.54)$ \\
\hline \multirow[t]{2}{*}{ VC } & 0.15 & 0.05 & 0.03 & & 0.19 & 0.11 & 0.09 \\
\hline & $(-0.09-0.37)$ & $(-0.18-0.28)$ & $(-0.19-0.24)$ & & $(0.01-0.36)$ & $(-0.07-0.29)$ & $(-0.09-0.27)$ \\
\hline \multirow[t]{2}{*}{ WM } & 0.40 & 0.33 & 0.30 & 0.66 & & 0.12 & 0.04 \\
\hline & $(0.14-0.61)$ & $(0.08-0.55)$ & $(0.07-0.51)$ & $(0.57-0.76)$ & & $(-0.05-0.29)$ & $(-0.12-0.22)$ \\
\hline \multirow[t]{2}{*}{ PO } & 0.10 & 0.01 & 0.35 & 0.61 & 0.72 & & 0.04 \\
\hline & $(-0.17-0.36)$ & $(-0.24-0.28)$ & $(0.11-0.57)$ & $(0.51-0.70)$ & $(0.60-0.82)$ & & $(-0.13-0.22)$ \\
\hline \multirow[t]{2}{*}{ PS } & 0.25 & 0.39 & 0.09 & 0.35 & 0.62 & 0.51 & \\
\hline & $(-0.02-0.50)$ & $(0.12-0.63)$ & $(-0.15-0.31)$ & $(0.23-0.47)$ & $(0.49-0.74)$ & $(0.37-0.64)$ & \\
\hline
\end{tabular}

factors to the phenotypic correlations. Analyses included simultaneous correction for the effects of sex, age or cohort on the individual scores. Table $3 \mathrm{a}$ lists the genetic (below diagonal) and environmental correlations from the full AECholesky model, Table $3 \mathrm{~b}$ gives the heritabilities of each variable - these are not thoroughly discussed as they have been discussed previously (Posthuma et al., 2001a; Posthuma et al., 2000; Baaré et al., 2001). As an illustration, Figure 1 shows MRI scans of four individuals belonging to an $\mathrm{MZ}$ and a $\mathrm{DZ}$ twin pair. Table 4 provides the path coefficients as estimated.

All inter-domain environmental correlations are statistically non-significantly different from zero (as judged from the 95\% CIs) and do not contribute to the observed correlation, except for the environmental correlation $(-0.71)$ between Cerebellar volume and Perceptual Organization. The contribution of environmental factors to the phenotypic correlation is $\sqrt{0.13} \times-0.71 \times \sqrt{0.32}=-0.14$, whereas the contribution of genetic factors to the phenotypic correlation is $\sqrt{0.87} \times 0.35 \times \sqrt{0.68}=0.27$. Thus the maximum likelihood estimate of the phenotypic correlation is $-0.14+$ $0.27=0.13$.

Note: Intra-domain correlations in normal text inter-domain correlations in bold. 


\section{Table 3b}

Maximum Likelihood Estimates of Heritabilities of Brain Volumes and WAIS III Dimensions.

\begin{tabular}{lc}
\hline & Heritability \\
\hline Whole brain gray matter volume & 0.82 \\
Whole brain white matter volume & 0.87 \\
Cerebellar volume & 0.87 \\
Verbal Comprehension & 0.84 \\
Working Memory & 0.65 \\
Perceptual Organization & 0.68 \\
Processing Speed & 0.63 \\
\hline
\end{tabular}

The observed correlation between perceptual Organization and Gray matter volume consisted of a genetic contribution (0.07) and an environmental contribution (0.05), both did not reach significance. The other inter-domain phenotypic correlations were all completely explained by an underlying common genetic factor.

Verbal Comprehension is not genetically associated with any of the three brain volumes, whereas Working Memory is genetically associated with all three brain volumes. Processing Speed is genetically related with white matter volume, but not with gray matter volume or cerebellar volume.
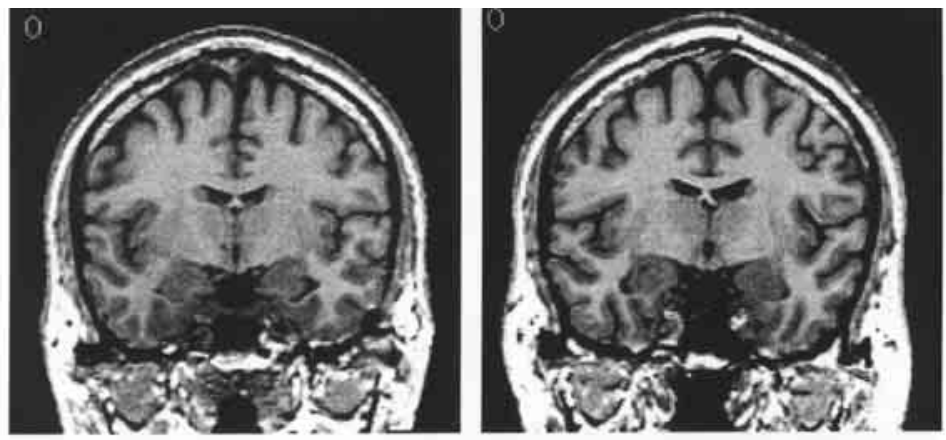

\section{MZ twins}
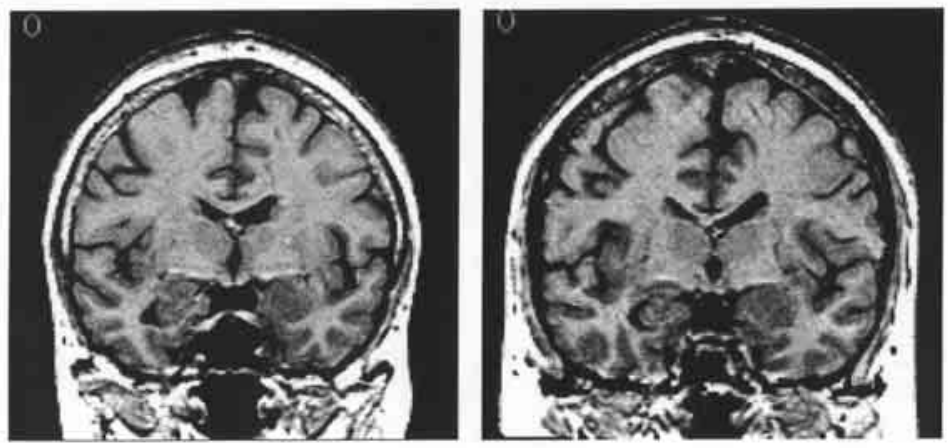

\section{DZ twins}

\section{Figure 1}

MR images of the brains of a same sex monozygotic twin pair (MZ twins, upper row) and a same sex dizygotic twin pair (DZ twins, lower row).

\section{Table 4}

Unstandardized Genetic and Non-shared Environmental Path Coefficients.

\begin{tabular}{|c|c|c|c|c|c|c|c|}
\hline & GMV & WMV & $\mathrm{CBV}$ & VC & WM & PO & PS \\
\hline \multicolumn{8}{|c|}{ Unstandardized Genetic Path Coefficients. } \\
\hline GMV & 47.93 & & & & & & \\
\hline WMV & 31.55 & 33.18 & & & & & \\
\hline CBV & 5.21 & 1.86 & 8.98 & & & & \\
\hline VC & 1.63 & -0.78 & -0.44 & 10.96 & & & \\
\hline WM & 4.19 & 0.86 & 1.11 & 6.60 & 6.97 & & \\
\hline PO & 0.91 & -0.75 & 3.38 & 5.65 & 3.66 & 5.11 & \\
\hline PS & 1.36 & 1.64 & -0.58 & 1.84 & 2.44 & 1.55 & 3.58 \\
\hline \multicolumn{8}{|c|}{ Unstandardized Non-shared Environmental Path Coefficients. } \\
\hline GMV & 22.54 & & & & & & \\
\hline WMV & 0.04 & 17.94 & & & & & \\
\hline CBV & 0.31 & 1.42 & 3.76 & & & & \\
\hline VC & -0.71 & 0.35 & -1.28 & 4.66 & & & \\
\hline WM & -1.01 & 0.20 & -0.40 & 1.23 & 7.58 & & \\
\hline PO & 1.19 & -1.42 & -4.36 & -0.19 & 0.78 & 4.14 & \\
\hline PS & 0.01 & -0.71 & 1.43 & 0.85 & 0.15 & 1.53 & 3.37 \\
\hline
\end{tabular}




\section{Discussion}

Phenotypic correlations between gray and white matter volume, and cerebellar volume on the one hand, and the four WAIS III dimensions (Verbal Comprehension, Working Memory, Perceptual Organization, and Processing Speed) on the other hand, indicated that part of the interindividual variance in IQ dimensions is shared with interindividual variance in brain volumes. The most consistent correlation was found between brain volume and Working Memory, which is generally considered a central part of intelligence (Kyllonen \& Chrystal, 1990). Gray and white matter and cerebellar volume relate equally strong to Working Memory. The correlations between Working Memory and all three brain volumes were completely mediated by a common underlying genetic factor. Seventeen per cent of the genetic variation in Working Memory can be accounted for by genes influencing these three brain volumes.

Intriguingly, the Verbal Comprehension dimension, which was the most heritable of all four WAIS III dimensions, did not correlate to any of the three brain volumes. Results from lesion and neuroimaging studies indicate that left temporal and frontocortical regions predominantly influence tasks that tap verbal comprehension. Thompson et al. (2001) reported the highest heritabilities (ranging from 95-100\%) for gray matter density in these linguistic regions. They specifically reported a higher heritability in the left temporal parietal region - comprising Wernicke's regions thought to be involved in language processing — than in the right linguistic region (Thompson et al., 2001). For the present study broad volumes were available, as opposed to voxel based data. We therefore choose not to incorporate hemispheric effects, also because we previously demonstrated the high correlation ( $>$.9) between left-right broad volumetric measurements and the absence of differential heritabilities for the two hemispheres (Baaré et al., 2001). In the present study, the absence of a (genetic) association of Verbal Comprehension with global volumes of white and gray matter does not preclude the existence of a genetic association with more localized volumes of the brain, such as Wernicke's area or the dorsolateral prefrontal areas (Brodmann areas 9 and 46; Rajkowska \& Goldman-Rakic, 1995).

Processing Speed was genetically related to white matter volume. White matter volume includes all myelinated axons in the cerebrum. Thickness of the myelin sheath is related to nerve conduction velocity and therefore its relation to Processing Speed seems intuitively appealing. We previously showed that genetic variation in perceptual speed as indexed by inspection time accounted for only $10 \%$ of the genetic variance in Verbal IQ but for $22 \%$ of the genetic variance in Performance IQ (Posthuma et al., 2001b). As perceptual speed is likely to depend on axonal myelination, it can be hypothesized that part of the genes that influence IQ are common to the genes that influence myelination of axons by oligodendrocytes. A candidate gene known to be involved in myelination is the Plp gene (Boison \& Stoffel, 1994; Griffiths et al., 1995, Ikenaka \& Kagawa, 1995; Lemke, 1993). Other genes implicated to be important for myelination from knock out mouse studies are the cgt-gene (Stoffel and Bosio, 1997), the $M A G$ gene (Fujita et al., 1998, Sheikh et al., 1999; Bartsch, 1996 for a review), and the tn-r gene (Weber et al., 1999).

Part of the genes responsible for cerebellar volume are also responsible for Perceptual Organizational ability, as reflected by the genetic correlation between these two measures. Although traditionally the cerebellum has been viewed as a neural substrate mainly involved in motor control (e.g., Ito, 1984), the presence of a correlation between cerebellar volume and components of intelligence has been reported since the 1980s (e.g., Leiner et al., 1986, 1993). Functional neuroimaging studies have shown that the cerebellum is involved in both motor and non-motor cognitive operations, such as working memory (Klingberg et al., 1996), complex problem solving (Kim et al., 1994), attentional activation (Allen et al., 1997), and semantic association (Petersen et al., 1989; Martin et al., 1995). The involvement of the cerebellum in higher cognitive functions may not be surprising from a biophysiological point of view, as the human cerebellum contains more neurons than the remainder of the brain combined (Williams \& Herrup, 1988), and has axonal connections with all major subdivisons of the central nervous system.

The genetic association of cerebellar volume with both Perceptual Organization and Working Memory is indicative of its general role in cognition. Recently, Airey et al. (2001) reported linkage of five quantitative trait loci for cerebellar size in mice, and proposed a set of candidate genes lying within the linkage regions. For example, the Pax 2 gene on chromosome 19 plays a critical role in early development of the cerebellum. Human homologous chromosomal regions of the five QTL's in mice as reported by Airey et al. (2001), are 1q23-43, 10q11-23, 9q13-q24, 11q12-q13, 10q23-qter, 16q12-22. These regions may also contain candidate genes for cognition.

The recent advances in morphometric/imaging techniques will enable future research to investigate functionvolume relations on a voxel based manner (e.g., Ashburner \& Friston, 2000; Hulshoff Pol et al., 2001). This may eventually lead to a detailed map of the human brain as it relates to specific cognitive abilities. The present study has made a first step in this direction by determining that the long-known relation between brain size and intelligence, even if using relatively unrefined measures such as total gray matter, total white matter, or total cerebellar volume, is of genetic origin.

Some caution in interpretation of a "genetic association" must be taken: determining that the association between different brain volumes and WAIS III-IQ dimensions is of genetic origin, as opposed to being of environmental origin, does not resolve the direction of causation between these two domains of measures. Four scenarios may underlie the established genetic association: 1) pleiotropy - there is a set of genes that influences both brain volumes and scores on the WAIS III dimensions; 2) unidirectional causation there is a set of genes that influences variation in brain volumes and this variation in turn leads to variation in WAIS III dimension scores; 3) reversed unidirectional causation - there is a set of genes that influences variation in WAIS III dimension scores and this variation in turn leads 
to variation in brain volumes; 4) reciprocal causation a combination of scenarios 2 and 3 .

Which of these four scenarios is most plausible has not been resolved yet, we merely established that the association follows a genetic pathway. Future studies will need to resolve the direction of causation to understand the plasticity of the brain and its role in cognition.

\section{References}

Airey, D. C., Lu, L., \& Williams, R. W. (2001). Genetic control of the mouse cerebellum: Identification of quantitative trait loci modulating size and architecture. Journal of Neuroscience, 21(14), 5099-5109.

Allen, G., Buxton, R. B., Wong, E. C., Courchesne, E. (1997). Attentional activation of the cerebellum independent of motor involvement. Science, 275(5308), 1940-1943.

Andreasen, N. C., Flaum, M., Swayze, V. 2nd, O’Leary, D. S., Alliger, R., Cohen, G., et al. (1993). Intelligence and brain structure in normal individuals. American Journal of Psychiatry, 150(1), 130-134.

Ashburner, J., \& Friston, K. J. (2000). Voxel-based morphometry: The methods. Neuroimage, 11, 805-821.

Baaré, W. F. C (2001). Brain structure abnormalities in schizophrenia and genetic variation in human brain morphology. Unpublished doctoral dissertation. University of Utrecht, The Netherlands.

Baaré, W. F. C., Hulshoff Pol, H. E., Boomsma, D. I., Posthuma, D., Geus, E. J. C, de, Schnack, H. G., et al. (2001). Quantitative genetic modeling of human brain morphology variation. Cerebral Cortex, 11, 816-824.

Bartley, A. J., Jones, D. W., \& Weinberger, D. R. (1997). Genetic variability of human brain size and cortical gyral patterns. Brain, 120, 257-269

Bartsch, U. (1996). Myelination and axonal regeneration in the central nervous system of mice deficient in the myelin-associated glycoprotein. Journal of Neurocytology, 25, 303-313.

Boison, D., \& Stoffel, W. (1994). Disruption of the compacted myelin sheath of axons of the central nervous system in proteolipid protein-deficient mice. Proceeding of the National Academy of Science, 91(24), 11709-11713.

Boomsma, D. I. (1998). Twin registers in Europe: An overview. Twin Research, 1(1), 34-51.

Bouchard, T. J. Jr., \& McGue, M. (1981). Familial studies of intelligence: A review. Science, 212, 1055-1059.

Carmelli, D., DeCarli, C., Swan, G. E., Jack, L. M., Reed, T., Wolf, P. A., et al. (1998). Evidence for genetic variance in white matter hyperintensity volume in normal elderly male twins. Stroke, 29, 1177-1181

Carmelli, D., Swan, G. E., DeCarli, C., \& Reed, T. (2002). Quantitative genetic modeling of regional brain volumes and cognitive performance in older male twins. Biological Psychology, 61(1-2), 139-155.

Deary, I. J. (2001). Human intelligence differences: A recent history. Trends in Cognitive Science, 5, 127-130.

Egan, V., Chiswick, A., Santosh, C., Naidu, K., Rimmington, J. E., \& Best, J. J. K. (1994). Size isn't everything - A study of brain volume, intelligence and auditory-evoked potentials. Personality and Individual Differences, 17(3), 357-367
Fujita, N., Kemper, A., Dupree, J., Nakayasu, H., Bartsch, U., Schachner, M., et al. (1998). The cytoplasmic domain of the large myelin-associated glycoprotein isoform is needed for proper CNS but not peripheral nervous system myelination. Journal of Neuroscience, 15(6), 1970-1978.

Griffiths, I. R., Montague, P., \& Dickinson, P. (1995). The proteolipid protein gene. Neuropathology and Applied Neurobiology, $21,85-96$.

Hulshoff Pol, H. E., Schnack, H. G., Mandl, R. C. W., van Haren, N. E. M., Koning, H., Collins, D. L., et al. (2001). Focal gray matter density changes in schizophrenia. Archives of General Psychiatry, 58, 1118-1125.

Ikenaka, K., \& Kagawa, T. (1995). Transgenic systems in studying myelin gene expression. Developmental Neuroscience, 17, 127-136.

Ito, M. (1984). The cerebellum and neural control. New York: Raven Press.

Jensen, A. R. (1994). Psychometric g related to differences in head size. Personality and Individual Differences, 17, 597-606.

Kim, S. G., Ugurbil, K., \& Strick, P. L. (1994). Activation of a cerebellar output nucleus during cognitive processing. Science, 12(265), 949-951.

Klingberg, T., Kawashima, R., \& Roland, P. E. (1996). Activation of multi-modal cortical areas underlies short-term memory. European Journal of Neurosciences, 8(9), 1965-1971.

Kyllonen, P. C., \& Christal, R. E. (1990). Reasoning ability is (little more than) working-memory capacity? Intelligence, 14, 389-433.

Leiner, H. C., Leiner, A. L., \& Dow, R.S. (1993). Cognitive and language functions of the human cerebellum. Trends in Neuroscience, 16(11), 444-447.

Leiner, H. C., Leiner, A. L., \& Dow, R. S. (1986). Does the cerebellum contribute to mental skills? Behavioral Neuroscience, 100(4), 443-454.

Lemke, G. (1993). The molecular genetics of myelination: An update. Glia, 7, 263-271.

Martin, A., Haxby, J. V., Lalonde, F. M., Wiggs, C. L., \& Ungerleider, L. G. (1995). Discrete cortical regions associated with knowledge of color and knowledge of action. Science, 270, 102-105.

McClearn, G. E., Johansson, B., Berg, S., Pedersen, N. L., Ahern, F., Petrill, S., et al. (1997). Substantial genetic influence on cognitive abilities in twins 80 or more years old. Science, 276, 1560-1563.

Neale, M. C. (1997). Mx: Statistical modeling (3rd ed.). Box 980126 MCV, Richmond VA 23298.

Pennington, B. F., Filipek, P. A., Lefly, D., Chhabildas, N., Kennedy, D. N., Simon, J. H., et al. (2000). A twin MRI study of size variations in human brain. Journal of Cognitive Neuroscience, 12(1), 223-232.

Petersen, S. E., Fox, P. T., Posner, M. I., Mintun, M. A., \& Raichle, M. E. (1989). Positron emission tomographic studies of the processing of single words. Journal of Cognitive Neuroscience, 1, 153-170.

Posthuma, D. (2002). Genetic variation and cognitive ability. Unpublished doctoral dissertation. Vrije Universiteit Amsterdam, The Netherlands. 
Posthuma, D., Geus, E. J. C. de, Baaré, W. F. C., Hulshoff Pol, H. E., Kahn, R. S., \& Boomsma, D. I. (2002). The association between brain volume and intelligence is of genetic origin. Nature Neuroscience, 5(2), 83-84.

Posthuma, D., Geus, E. J. C. de, \& Boomsma, D. I. (2001b). Perceptual speed and IQ are associated through common genetic factors. Behavior Genetics, 31(6), 593-602.

Posthuma, D., Geus, E. J. C. de, Neale, M. C., Hulshoff Pol, H. E., Baaré, W. F. C., Kahn, R. S., et al. (2000). Multivariate genetic analysis of brain structure in an extended twin design. Behavior Genetics, 30, 311-319.

Posthuma, D., Neale, M. C., Boomsma, D. I., \& Geus, E. J. C. de. (2001a). Are smarter brains running faster? Heritability of alpha peak frequency, IQ and their interrelation. Behavior Genetics, 31(6), 567-579.

Rajkowska, G., \& Goldman-Rakic, P. S. (1995). Cytoarchitectonic definition of prefrontal areas in the normal human cortex: II. Variability in locations of areas 9 and 46 and relationship to the Talairach Coordinate System. Cerebral Cortex, 5(4), 323-337

Raz, N., Torres, I. J., Spencer, W. D., Millman, D., Baertschi, J. C., \& Sarpel, G. (1993). Neuroanatomical correlates of agesensitive and age-invariant cognitive-abilities - An in-vivo MRI investigation. Intelligence, 17(3), 407-422.

Reveley, A. M, Reveley, M. A, Chitkara, B., \& Clifford, C. (1984). The genetic basis of cerebral ventricular volume. Psychiatry Research, 13, 261-266.

Schnack, H. G., Hulshoff Pol, H. E., Baaré, W. F. C., Staal, W. G., Viergever, M. A., \& Kahn, R. S. (2001a). Automated separation of gray and white matter from MR images of the human brain. NeuroImage, 13, 230-237.

Schnack, H. G., Hulshoff Pol, H. E, Baare, W. F. C., Viergever, M. A., \& Kahn, R. S. (2001b). Automatic segmentation of the ventricular system from MR images of the human brain. NeuroImage, 14, 95-104.

Sheikh, K. A., Sun, J., Liu, Y., Kawai, H., Crawford, T. O., Proia, R. L., et al. (1999). Mice lacking complex gangliosides develop Wallerian degeneration and myelination defects. Proeedings of the National Academy of Science, 96(13), 7532-7537.
Stoffel, W., \& Bosio, A. (1997). Myelin glycolipids and their functions. Current Opinion in Neurobiology, 7(5), 654-661.

Storfer, M. (1999). Myopia, intelligence, and the expanding human neocortex: Behavioral influences and evolutionary implications. International Journal of Neuroscience, 98, 153-276.

Talairach, J., \& Tournoux, P. (1988). Co-Planar stereotaxic atlas of the human brain. Stuttgart, Germany: Georg Thieme Verlag.

Thompson, P. M., Cannon, T. D., Narr, K. L., van Erp, T., Poutanen, V. P., Huttunen, M., et al. (2001). Genetic influences on brain structure. Nature Neuroscience, 4(12), 1253-1258.

van Valen, L. (1974). Brain size and intelligence in man. American Journal of Physical Anthropology, 40(3), 417-423.

WAIS-III. (1997). Dutch version. Manual. Lisse: Swets and Zeitlinger.

Weber, P., Bartsch, U., Rasband, M. N., Czaniera, R., Lang, Y., Bluethmann, H., et al. (1999). Mice deficient for tenascin$\mathrm{R}$ display alterations of the extracellular matrix and decreased axonal conduction velocities in the CNS. Journal of Neuroscience, 19, 4245-4262.

Wickett, J. C., Vernon, P. A., \& Lee, D. H. (2000). Relationships between factors of intelligence and brain volume. Personality and Individual Differences, 29, 1095-1122.

Willerman, L., Schultz, R., Rutledge, J. N., et al. (1992). Hemisphere size asymmetry predicts relative verbal and nonverbal intelligence differently in the sexes - An MRI study of structure-function relations. Intelligence, 16(3-4), 315-328.

Williams, R. W., \& Herrup, K. (1988). The control of neuron number. Annual Review of Neuroscience, 11, 423-453.

Woodward, K., \& Malcolm, S. (1999). Proteolipid protein gene: Pelizaeus-Merzbacher disease in humans and neurodegeneration in mice. Trends in Genetics, 15(4), 125-128.

Wright, M. J., Boomsma, D. I., Geus, E. J. C. de, Posthuma, D., Van Baal, G. C. M., Luciano, M., et al. (2001). Genetics of cognition: Outline of collaborative twin study. Twin Research, 4, 48-56. 